\title{
Amplification of surface acoustic waves by transverse electric current in piezoelectric semiconductors
}

\section{Gulyaev, Yuri V.}

\section{Published in:}

Applied Physics Letters

Link to article, DOI:

$10.1063 / 1.1655236$

Publication date:

1974

\section{Document Version}

Publisher's PDF, also known as Version of record

Link back to DTU Orbit

Citation (APA):

Gulyaev, Y. V. (1974). Amplification of surface acoustic waves by transverse electric current in piezoelectric semiconductors. Applied Physics Letters, 24(9), 405-406. https://doi.org/10.1063/1.1655236

\section{General rights}

Copyright and moral rights for the publications made accessible in the public portal are retained by the authors and/or other copyright owners and it is a condition of accessing publications that users recognise and abide by the legal requirements associated with these rights.

- Users may download and print one copy of any publication from the public portal for the purpose of private study or research.

- You may not further distribute the material or use it for any profit-making activity or commercial gain

- You may freely distribute the URL identifying the publication in the public portal 


\title{
Amplification of surface acoustic waves by transverse electric current in piezoelectric semiconductors
}

\author{
Yuri V. Gulyaev* \\ Physics Laboratory III, Technical University of Denmark, Lyngby, Denmark \\ (Received 28 September 1973) \\ It is shown that the principal characteristic feature of the surface acoustic waves in piezoelectrics- \\ the presence of an alternating electric field transverse to the surface, which can be of the same order \\ of magnitude as the longitudinal field-may not only give rise to the known transverse \\ acoustoelectric effect but also lead to amplification of surface acoustic waves by electron drift \\ perpendicular to the surface. For Love waves in a piezoelectric semiconductor film on a highly \\ conducting substrate, the amplification coefficient is found and the conditions necessary for \\ amplification are investigated.
}

The first publication containing the idea of the possibility of amplification of surface acoustic waves (SAW) by the drift of electrons in solids, in particular in the layered piezoeielectric-semiconductor structures (LPDS), and the theory of this phenomenon for the simplest case of Love waves in a structure of a piezoelectric film on a semiconducting substrate (or vice versa) was made by Gulyaev and Pustovoit $^{1}$ (see also Ref. 2). The first experimental observations of the effect both in uniform piezoelectric semiconductors ${ }^{3,4}$ and in LPDS structures ${ }^{5-7}$ showed that the amplification of SAW may be quite large and can be obtained in a $\mathrm{cW}$ regime. During recent years there was quite a broad interest in studying the interaction of SAW with electrons not only because of the practical aspects of the possibility of their amplification, their accessibility, and the compatibility of their technology with the usual integrated circuit technology, but also because of a number of new physical effects specific for SAW only. In this paper we consider one such new effect-the possibility of amplification of SAW by an electric current directed transverse to the surface along which the SAW propagates. The possibility of amplification of SAW when the drift electric field is perpendicular to the propagation direction of the wave is based on the physical concept of the amplification of sound by drifting electrons in solids. For example, in the "hydrodynamic" case $q l \ll I$ ( $q$ is the acoustic wave number, $l$ is the mean free path of electrons), the amplification of bulk acoustic waves (BAW) (and SAW by longitudinal current) is due to the bunching of electrons in the longitudinal direction and to the creation by the external electric (also longitudinal) field of such a condition that the alternating longitudinal electric field of the wave performs negative work on the electrons in the bunches. In fact the amplification starts when the phase shift between the maxima of electron density and the corresponding minima of the potential energy of electrons in the sound wave in the direction of its propagation changes from negative (retardation) to positive (advancement).

The Rayleigh or Love SAW is principally different from the BAW in the sense that it is accompanied (in piezoelectrics) by an alternating electric field trans verse to the surface of the same order of magnitude as the longitudinal alternating field. So there is bunching of electrons not only in the longitudinal direction, but also in the direction transverse to the surface. In a piezoelectric semiconductor this bunching is such that in the absence of the external transverse electric current the maxima of the electron density lie a little bit further from the surface than the corresponding minima of the potential energy of electrons in the sound wave in this transverse direction. This means that there exists a de force acting on the bunched electrons which is directed towards the surface. This force gives rise to a transverse acoustoelectric current or electromotive force ("transverse acoustoelectric effect") which was predicted and experimentally observed earlier. ${ }^{8-10}$ Following the same sort of arguments as in the case of amplification of SAW by longitudinal current, one may assume that by applying a strong enough electric field in the direction transverse to the surface it is possible to shift the electron bunches in this direction to the opposite slope of the potential wells created by the SAW as mentioned above. In complete analogy with the longitudinal case it would mean that the transverse alternating field of the SAW will perform negative work on the bunched electrons and the SAW itself will be amplified.

These qualitative arguments are confirmed by a calculation again for the simplest case of Love waves in a CdS film with $c$ axis parallel to the surface on the highly conductive (isotropic or cubic, say $\mathrm{Ge}$ ) substrate with polarization along the $c$ axis. The top of the film is metallized and serves as one contact for applying the transverse drift electric field while the substrate itself serves as the other contact. The calculation is quite analogous to that for the longitudinal case. ${ }^{8}$ One solves the linearized system of equations - two-dimensional sound propagation equation, Poisson equation, the continuity equation together with the phenomenological expressions for the electric current components - and then using proper boundary conditions one finds the dispersion equation from which the absorption (amplification) coefficient is calculated. The expression for the amplification coefficient under the simplifying condi tions $q r_{D} \ll 1, q h \gtrsim 1$, and $\kappa \tau_{M} v_{d} \simeq 1\left(r_{D}\right.$ and $\tau_{M}$ are the Debye length and Maxwellian relaxation time, $\omega$ the frequency of the wave, $h$ the thickness of the film, $v_{d}$ the electron drift velocity, and $\kappa$ a constant of the order of $q$ which determines the change of the amplitude of the wave in the transverse direction) has the form

$$
\alpha_{e}=\zeta q \frac{\omega \tau_{M}\left(F_{1}-F_{2} \kappa_{M} v_{d}\right)}{\left|1+\left(\omega-\kappa v_{d}\right)^{2} \tau_{M}^{2}\right|^{2}}
$$

Here $\zeta$ is the electromechanical coupling constant and $F_{1}$ and $F_{2}$ are the positive quantities of the order of unity which depend on the geometry of the structure, on 
the mechanical properties of the materials, and on the wave frequency. So the criterion for amplification of SAW by transverse drift of electrons is

$$
v_{d}>\frac{F_{1}}{F_{2}} \frac{1}{\kappa \tau_{M}}
$$

and may require approximately the same electric field as that in the case of amplification by a longitudinal current. It is obvious that the Rayleigh SAW also can be amplified by the transverse drift of electrons, but the calculation of the effect is a bit more cumbersome and will be published elsewhere.

Let us note finally that the SAW in the high-frequency case $q l \gg 1$ also may be amplified by the transverse drift of electrons since there is the possibility of momentum transfer from the surface wave to electrons in the direction transverse to the surface. ${ }^{11}$ To calculate this effect one must consider the exchange of momentum and energy between the surface phonons (of which the SAW consists) and the electrons whose distribution is shifted by the transverse electric current.

The author would like to thank Professor N.I. Meyer and Dr. E. Mosekilde for helpful discussion of the work.
* Permanent address: Institute of Radio Engineering and Electronics, Academy of Science, Moscow, USSR.

${ }^{1}$ Yu. V. Gulyaev, V.I. Pustovoit, Zh. Eksp. Teor. Fiz. 47, 2251 (1964) [Sov. Phys. -JETP 20, 1508 (1965)].

${ }^{2}$ Amplification of longitudinal acoustic waves by the drift of electrons in a surface layer was considered by Kalissky [S. Kalissky, Proceedings of Conference on Vibration Problems, Warsaw, Vol. 6, 323 (1965) (unpublished) ]; P. K. Tien presented analogous ideas in US Patent No. 3158819 (1964).

${ }^{3}$ R. M. White, T.W. Voltmer, Appl. Phys. Lett. 8, 40 (1966).

${ }^{4}$ V. I. Vaskova, I. A. Viktorov, Acoust. Zh. 13, 292 (1967)

[Sov. Phys. -Acoust. 13, 249 (1967)].

${ }^{5}$ K. Yoshida and M. Yamanishi, Jap. J. Appl. Phys. 7, 1143 (1968).

${ }^{6}$ J. H. Collins, K. M. Lakin, C. F. Quate, and H. J. Shaw, Appl. Phys. Lett. 13, 314 (1968).

${ }^{\text {?}}$ Yu. V. Gulyaev, A. M. Kmita, I. M. Kotelyansky, A. V. Medved, and Sh.S. Tursunov, Fiz. Tekh. Poluprovodn. 5, 80 (1971) [Sov. Phys. -Semicond. 5, 66 (1971).

${ }^{8}$ Yu. V. Gulyaev, A. Yu. Karabanov, A. M. Kmita, A. V. Medved, and Sh. S. Tursunov, Fiz. Tverd. Tela 12, 2595 (1970) [Sov. Phys. -Solid State 12, 2085 (1971)].

${ }^{9}$ A. M. Kmita and A.V. Medved, JETP Lett. 14, 455 (1971).

${ }^{10}$ In the case of a LPDS structure it is easy to show that the transverse dc force acting on the bunched electrons is directed from the interface between the semiconductor and piezoelectric layers towards the inside of a semiconductor layer.

${ }^{11} \mathrm{This}$ momentum transfer must produce also the transverse acoustoelectric effect.

\title{
Harmonic generation and frequency mixing in laser-produced plasmas*
}

\author{
Ping Lee', D. V. Giovanielli, R. P. Godwin, and G. H. McCall \\ Los Alamos Scientific Laboratory, University of California, Los Alamos, New Mexico 87544 \\ (Received 26 December 1973)

\begin{abstract}
Spectral measurements of light scattered at various angles from a laser-produced plasma were made at the laser frequency $\omega_{L}$, and at $\omega_{L} / 2,3 \omega_{L} / 2,2 \omega_{L}, 5 \omega_{L} / 2$, and $3 \omega_{L}$ using a mode-locked Nd:glass laser to produce intensities of $10^{16} \mathrm{~W} / \mathrm{cm}^{2}$ at the target. Signals were detected at $\omega_{L}, 3 \omega_{L} / 2$, and $2 \omega_{L}$, and their intensities relative to the incident intensity were determined. Upper bounds were set
\end{abstract} \\ for the intensities at the other frequencies.
}

Various investigator $\mathrm{s}^{1-7}$ have reported measurements of scattered light from plasmas created by a focused 1.06- $\mu \mathrm{m}$ wavelength laser. Such studies are of vital interest to the laser-fusion program. Plasma-light interactions (such as stimulated Brillouin, Raman, or Compton scattering) can, in principle, lead to enhanced target reflectance and thus poor energy coupling. ${ }^{8}$ With incident laser intensities of $10^{14}-10^{15} \mathrm{~W} / \mathrm{cm}^{2}$ and pulse lengths of $1-3 \mathrm{nsec}$, scattered light has been observed at the laser frequency $\omega_{L}$ and at the frequencies $2 \omega$ and $\frac{3}{2} \omega_{L}$. In one case $e^{3}$ measurable signals were reported at $\frac{1}{2} \omega_{L}$.

We have made spectral measurements of light scattered at various angles from targets irradiated with a short-pulse (25 psec) Nd:YAG-Nd:glass laser. The incident laser light was focused to about a $50 \mu \mathrm{m}$ diameter on the target with an $f / 3.5$ lens. Intensities in the region of the focal spot were greater than $10^{16} \mathrm{~W} / \mathrm{cm}^{2}$. The spectral width of the incident light was approximate- ly $1 \AA$. The scattered light spectra were analyzed with a Mc Pherson model $2161-\mathrm{m}$ spectrometer which has a resolution of less than $0.5 \AA$.

The high incident power used in these experiments is believed to be above the threshold for stimulated processes. ${ }^{8}$ The narrow incident linewidth (some of the earlier experiments have been performed with a $30-\AA-$ wide incident line) and short well-characterized pulses of the laser radiation may simplify interpretation of the experimental results.

Two types of targets were used in the experiments: gold wires of $50-\mu \mathrm{m}$ diameter and plane polyethylene $\left(\mathrm{CH}_{2}\right)$ sheets about $1 \times 10^{-2} \mathrm{~cm}$ thick. The gold wires intercepted more than $80 \%$ of the incident laser light. (This measurement verifies that most of the focused radiation falls within a $50-\mu \mathrm{m}$ diameter.) The wires exhibit specular scattering at all angles in the plane normal to the wire axis. Plane targets were tipped so 